\title{
POLARITIES AND OVALS IN THE HUGHES PLANE
}

\author{
T. G. ROOM
}

(Received 18 July 1969)

Summary. In 1946 Baer (Polarities in finite projective planes, Bull. Am. Math. Soc. 52, 77-93) showed that the absolute points of a polarity in a finite projective plane of odd non-square order always form an oval, that is, in a plane of order $n$ there are exactly $n+1$ absolute points and no three are collinear. It is well known that the absolute points of polarities in planes of odd square order form ovals in some cases.

If the oval is a subset of the set of absolute points, then the oval itself determines the polarity, and this makes it appear unlikely that the oval could be a proper subset. Among other results in the paper it is to be proved that in the regular Hughes plane there is a polarity which is determined by an oval which is a relatively small subset of the set of absolute points. Explicitly, if $\Omega$ is the Hughes plane of order $q^{2}$ and $\Delta$ is the central subplane of order $q$, then every conic in $\Delta$ can be extended to an oval in $\Omega$, and this oval determines a polarity in which there are $\frac{1}{2}\left(q^{3}-q\right)$ additional absolute points.

\section{Algebraic preliminaries}

$F: G F(q), q$ odd; elements of $F$ : lower case italic letters.

$F_{S}, F_{N}$ : the sets respectively of squares and not-squares in $F$.

$\Phi: G F\left(q^{2}\right)$; elements of $\Phi$ : lower case Greek letters (except $(x, y, z)$ for coordinates). $\alpha=a+v a^{\prime}, \alpha^{*}=a-v a^{\prime}=\alpha^{q}, v^{2}=u, u \in F_{N} . d_{\alpha}=a^{2}-u a^{\prime 2}=$ $\alpha^{q+1}$.

$\Phi_{S}, \Phi_{N}: \alpha \in \Phi_{S}$ or $\Phi_{N}$ according as $d_{\alpha} \in F_{S}$ or $F_{N}$. Subscripts $S$ and $N$ will be used to designate the subset to which an element belongs, thus $\alpha_{S}: \alpha \in \Phi_{S}$, $\alpha_{N}: \alpha \in \Phi_{N}$. For all $\rho, \rho^{2} \in \Phi_{S}$.

$\Psi$ : the Dickson near-field with the same elements as $\Phi$, in which addition (and multiplication by members of $F$ ) are identical with the corresponding operations in $\Phi$, and, in an obvious notation, multiplication is defined by:

$$
\left(\rho \sigma_{S}\right)_{\Psi}=(\rho \sigma)_{\Phi},\left(\rho \sigma_{N}\right)_{\Psi}=\left(\rho^{*} \sigma\right)_{\Phi} .
$$

From these definitions we deduce the following product-relations in $\Psi$ :

$$
\begin{aligned}
\left(\rho \sigma_{S}^{-1}\right)_{\Psi} & =\left(\rho \sigma^{-1}\right)_{\Phi}, & \left(\rho \sigma_{N}^{-1}\right)_{\Psi} & =\left(\rho^{*} \sigma^{*-1}\right)_{\Phi} . \\
\rho_{S}^{-1} & =\rho_{S}^{*} / d_{\rho}, & \rho_{N}^{-1} & =\rho_{N} / d_{\rho} . \\
\rho_{S} \sigma_{S} & =\sigma_{S} \rho_{S}, & \rho_{S} \sigma_{N} & =\sigma_{N} \rho_{S}^{*}, \\
\rho_{N} \sigma_{S} & =\sigma_{S}^{*} \rho_{N}, & \rho_{N} \sigma_{N} & =\sigma_{N}^{*} \rho_{N}^{*} .
\end{aligned}
$$




$$
\begin{aligned}
\rho_{S} \rho_{S}^{*} & =\rho_{S}^{*} \rho_{S}=d_{\rho}, \\
\left(\rho_{N} \rho_{N}^{*}\right)_{\Psi} & =\left(\rho^{* 2}\right)_{\Phi}, \quad\left(\rho_{N}^{*} \rho_{N}\right)_{\Psi}=\left(\rho^{2}\right)_{\Phi} .
\end{aligned}
$$

The Hughes plane $\Omega$ over $\Psi$, Hughes [1] ${ }^{1}$, is defined thus:

Points: column-vectors $(x, y, z) .(x, y, z) \kappa$ is the same point for all $\kappa \neq 0$.

Real point $(x, y, z)$ : for som $2 \kappa$, each component of $(x, y, z) \kappa \in F$.

$\Delta$ : the subplane of order $q$ in $\Psi$ containing the real points.

Complex point : point of $\Omega-\Delta$.

Line of $\Omega:$ a set of points $(a, b, c) \cup\{(a, b, c) \kappa+(\rho, \sigma, \tau): \kappa \in \Psi\}$.

As an immediate consequence of this definition we have:

LemMa 1. $\boldsymbol{a} \in F, \boldsymbol{b} \in F$ are the coordinate-vectors of two distinct points of $\Delta$ and $\eta \in \Psi$ is a coordinate-vector of any point not collinear with $a, b$. Then the set of points $\{\boldsymbol{r a}+\boldsymbol{s} \boldsymbol{b}+\boldsymbol{t \eta}: \boldsymbol{r}, s, t \in F\}$ forms a subplane of order $q$ in $\Omega$.

The definition of a line, given above, is equivalent to that given by Ostrom [3], namely; a line is the set of points satisfying one of the following equations:

$$
\text { (i) } \begin{aligned}
y-s z & =\kappa(x-r z), r, s, m \in F, \kappa \in \Psi-F, \\
\text { (ii) } y & =m x+\lambda z, \lambda \in \Psi, \\
0 & =x+\lambda z ; z=0 .
\end{aligned}
$$

In general, results in this paper will be proved only for equations in the first form. Proofs in other relevant cases are straightforward adaptations.

\section{The extension of a conic in $\Delta$ to an oval in $\Omega$}

The group of projectivities of $\Delta$ extends to $\Omega$ (Rosati [4]) so that all nonsingular conics in the subplane $\Delta$ are equivalent in their relations to the complex points of $\Omega$. Take in $\Delta$ the conic

$$
\gamma: x y=z^{2},
$$

and define $\Gamma$ as the following set of points in $\Omega$ :

$$
\Gamma:\{(1,0,0),(0,1,0)\} \cup\left\{x z^{-1}=\left(z y^{-1}\right)_{S}\right\} \cup\left\{x z^{-1}=\left(z^{*} y^{*-1}\right)_{N}\right\} .
$$

$\Gamma$ contains $q^{2}+1$ points, $q+1$ real points forming $\gamma$, and $q^{2}-q$ complex points, forming the set

$$
\Gamma-\gamma:\left\{\left(\rho_{S}, \rho_{S}^{-1}, 1\right)\right\} \cup\left\{\left(\rho_{N}, \rho_{N}^{*-1}, 1\right), \rho \in \Psi-F\right\}=\left\{\left(\rho, \rho^{*} / d_{\rho}, 1\right)\right\} .
$$

Let $\boldsymbol{C}$ be any projectivity of the group $\mathscr{C}$ of projective automorphisms of $\gamma$ in $\Delta$ extended to $\Omega$, and let $C\left(\rho, \rho^{-1}, 1\right)=(\lambda, \mu, v)$. Then, since $(\lambda, \mu, v)$, considered

1 Hughes defines the plane in terms of a left-near-field; in this paper (following Hall [2]), we use a right-near-field. 
as a point of the Galois plane over $\Phi$, is a point of the conic $x y=z^{2}$ in that plane, we have

$$
\left(\lambda v^{-1}\right)_{\Phi}=\left(v \mu^{-1}\right)_{\Phi},
$$

and therefore, from relations (2),

$$
\left.\begin{array}{c}
\left(\lambda v_{S}^{-1}\right)_{\Psi} \\
\left(\lambda^{*} v_{N}^{*-1}\right)_{\Psi}
\end{array}\right\}=\left\{\begin{array}{l}
\left(v \mu_{S}^{-1}\right)_{\Psi} \\
\left(v^{*} \mu_{N}^{*-1}\right)_{\Psi} .
\end{array}\right.
$$

That is, for points of $\Omega, \lambda \nu^{-1}=\left(v \mu^{-1}\right)_{S}$ or $\left(v^{*} \mu^{*-1}\right)_{N}$.

THEOREM 1 The set of $q^{2}+1$ points:

$$
\left.\Gamma:\{(1,0,0),(0,1,0)\} \cup\left\{\rho_{S}, \rho_{S}^{-1}, 1\right)\right\} \cup\left\{\left(\rho_{N}, \rho_{N}^{*-1}, 1\right)\right\}, \rho \in \Psi
$$

is invariant under the extension to $\Omega$ of the projectivities of $\Delta$ that fix the conic $x y=z^{2}$ in $\Delta$.

In relation to $\gamma$, the points of $\Delta$ may be split into three subsets:

$\left\{P_{\gamma}\right\}$ : the vertices of $\gamma$, for which $z^{2}-x y=0$,

$\left\{P_{S}\right\}$ : the exterior points of $\gamma, z^{2}-x y \in F_{S}$,

$\left\{P_{N}\right\}$ : the interior points of $\gamma, z^{2}-x y \in F_{N}$

These three subsets are invariant under the operations of $\mathscr{C}$, and operations of $\mathscr{C}$ can be found which map any point of $\Delta$ to any point of the same subset. Consequently all points of each of the subsets $\left\{P_{\gamma}\right\},\left\{P_{S}\right\},\left\{P_{N}\right\}$ are equivalent in their relations to the complex set $\Gamma-\gamma$, and we can discuss the relation of vertices of $\Gamma-\gamma$ to points of $\Delta$ in terms of their relations to the three typical points:

$$
X=(1,0,0) \text { of }\left\{P_{\gamma}\right\}, Z=(0,0,1) \text { of }\left\{P_{S}\right\} \text { and } U=(-1, u, 0) \text { of }\left\{P_{N}\right\} \text {. }
$$

We are to prove:

THEOREM 2. If $Q$ is any vertex of $\Gamma-\gamma$ then:

(i) $X Q$ contains no other vertex,

(ii) $Z Q$ contains exactly one other vertex,

(iii) For two particular vertices $Q_{u}, Q_{u}^{\prime}$ the lines $U Q_{u}, U Q_{u}^{\prime}$ contain no other vertex, for every other vertex $Q$ the line $U Q$ contains exactly one other vertex.

Take $Q$ to be $\left(\rho_{S}, \rho_{S}^{-1}, 1\right)$ or $\left(\rho_{N}, \rho_{N}^{*-1}, 1\right)$.

(i) (a) A general point $K$ of $X Q$ is $\left(\kappa, \rho_{S}^{-1}, 1\right)$ or $\left(\kappa, \rho_{N}^{*-1}, 1\right) . K$ is a point of $\Gamma-\gamma$ only if $\kappa=\rho$; that is, $K=Q$.

(b) The tangent to $\gamma$ at $X$ is $y=0$; this line contains no point of $\Gamma-\gamma$.

(ii) A general point $K$ of $Z Q$ is $\left(\rho_{S}, \rho_{S}^{-1}, \kappa\right)$ or $\left(\rho_{N}, \rho_{N}^{*-1}, \kappa\right)$; to test whether $K \in \Gamma-\gamma$ we have to consider the following four cases:

(a) $\left(\rho_{S}, \rho_{S}^{-1}, \kappa_{S}\right): \rho_{S} \kappa_{S}^{-1}=\kappa_{S} \rho_{S}=\rho_{S} \kappa_{S} \Rightarrow \kappa_{S}^{2}=1$. 
Thus in this set there is one point $K, \neq Q$, namely $\left(\rho_{s}, \rho_{S}^{-1},-1\right)$, which belongs to $\Gamma-\gamma$.

(b) $\left(\rho_{S}, \rho_{S}^{-1}, \kappa_{N}\right): \rho_{S} \kappa_{N}^{-1}=\kappa_{N}^{*} \rho_{S}^{*}=\rho_{S} \kappa_{N}^{*}$ $\Rightarrow \kappa_{N}^{-1}=\kappa_{N}^{*} \Rightarrow \kappa_{N}^{*}=\kappa_{N} / d_{\boldsymbol{k}}$. No solution.

(a') $\left(\rho_{N}, \rho_{N}^{*-1}, \kappa_{N}\right): \rho_{N} \kappa_{N}^{-1}=\kappa_{N} \rho_{N}^{*}=\rho_{N} \kappa_{N}^{*}$. No solution.

(b') $\left(\rho_{N}, \rho_{N}^{*-1}, \kappa_{S}\right): \rho_{N} \kappa_{S}^{-1}=\kappa_{S}^{*} \rho_{N}=\rho_{N} \kappa_{S} \Rightarrow \kappa_{S}^{2}=1$. Again there is one point of $\Gamma-\gamma$ other than $Q$ on $Z Q$.

(iii) A general point $K$ of $U Q$ may be taken to be

$$
K=(\rho-\kappa) U+Q=\left(\kappa,\left[\rho^{-1} \text { or } \rho^{*-1}\right]+\rho u-\kappa u, 1\right) .
$$

Since $K \in \Gamma-\gamma \Rightarrow K=\left(\kappa,\left[\kappa^{-1}\right.\right.$ or $\left.\left.\kappa^{*-1}\right], 1\right)$, the first two coordinates must belong either both to $\Phi_{S}$ or both to $\Phi_{N}$.

(a) $\left(\kappa_{S}, \rho_{S}^{-1}+\rho_{S} u-\kappa_{S} u, 1\right) \in \Gamma-\gamma \Rightarrow \kappa^{-1}=\rho^{-1}+\rho u-\kappa u$

$$
\begin{aligned}
\Rightarrow \kappa^{-1}-\rho^{-1} & =(\rho-\kappa) u \\
\Rightarrow(\rho-\kappa) u & =\left(1-\rho_{S}^{-1} \kappa_{S}\right) \kappa_{S}^{-1}=\left(1-\kappa_{S} \rho_{S}^{-1}\right) \kappa_{S}^{-1} \\
& =\left(\rho_{S}-\kappa_{S}\right) \kappa_{S}^{-1} \rho_{S}^{-1} \Rightarrow \kappa=\rho, \text { or } \kappa=u^{-1} \rho^{-1} .
\end{aligned}
$$

The line $U Q$ therefore contains exactly two points of $\Gamma-\gamma$ unless

But

$$
\rho_{s}=u^{-1} \rho_{S}^{-1}, \text { that is, } \rho_{S}^{2}=u^{-1} \text { or } \rho_{S}= \pm v u^{-1} \text {. }
$$

$$
v \in \Phi_{S} \Rightarrow-u \in F_{S} \Rightarrow-1 \in \Phi_{N} \Rightarrow q \equiv-1(\bmod 4) .
$$

That is, there are two lines through $U$ each containing only one point of $\Gamma-\gamma$ provided $q \equiv-1(\bmod 4)$. If $q=1(\bmod 4)$ there is no such line.

The cases

(b) $\left(\kappa_{N}, \rho_{S}^{-1}+\rho u-\kappa u, 1\right) \in \Gamma-y$,

(a') $\left(\kappa_{N}, \rho_{N}^{*-1}+\rho u-\kappa u, 1\right) \in \Gamma-\gamma$ and

(b') $\left(\kappa_{S}, \rho_{N}^{*-1}+\rho u-\kappa u, 1\right) \in \Gamma-\gamma$

can be analysed similarly and lead to corresponding results. In particular in case $\left(\mathrm{a}^{\prime}\right)$ we find there are two solutions $\kappa=\rho$ and $\kappa^{*}=u^{-1} \rho^{-1}$. These coincide when

$$
u^{-1}=\left(\rho_{N} \rho_{N}^{*}\right)_{\Psi}=\left(\rho^{* 2}\right)_{\Phi} \Rightarrow \rho_{N}= \pm v u^{-1} \text { when } q \equiv 1(\bmod 4)
$$

and otherwise there is no coincident pair.

This completes the proof of Theorem 2 . Since $X, Z, U$ can be transformed into respectively any points of the sets $\left\{P_{\gamma}\right\},\left\{P_{S}\right\},\left\{P_{N}\right\}$, it follows that any line through a point of $\Delta$ which contains one vertex of $\Gamma$, either contains only that vertex or it contains altogether exactly two vertices. But every line of $\Omega$ contains at least one point of $\Delta$, so that every line of $\Omega$ intersects the set $\Gamma$ in no point or one point or two points. We have proved that every tangent of $\gamma$ contains no other point of $\Gamma$, 
and that through $U$, and therefore through each point of the set $\left\{P_{N}\right\}$ in $\Delta$, there are two lines each containing only one point of $\Gamma$. The number of points in $\left\{P_{N}\right\}$ is $\frac{1}{2}\left(q^{2}-q\right)$ and the number of points in $\Gamma-\gamma$ is the number of elements of $\Psi-F$, namely, $q^{2}-q$, consequently there is a single line through any point of $\Gamma-\gamma$ which contains no other point of $\Gamma$. We have proved therefore that $\Gamma$ is a set of $q^{2}+1$ points in $\Omega$ with no three collinear, that is:

THEOREM 3. The set $\Gamma$ is an oval.

Consider now the set of vertices of $\Gamma$ for which $\rho=h v, h \in F$. Since $u=\left(v^{2}\right)_{\Phi}=$ $\left(v_{S} v_{S}\right)_{\Psi}=\left(v_{N}^{*} v_{N}\right)_{\Psi}$, the coordinate-vectors are $\left(h^{2} u, 1, h v\right)$. The set

$$
\gamma_{1}=(1,0,0) \cup\left\{\left(h^{2} u, 1, h v\right): h \in F\right\}
$$

is a subset of the set $\{l(1,0,0)+m(0,1,0)+n(0,0, v)\}$ and consequently, by Lemma 1, all points of $\gamma_{1}$ lie in a subplane, $\Delta_{1}$, say, of order $q$. Also $\gamma_{1}$, since it is a subset of $\Gamma$, consists of $q+1$ points no three collinear, so that the set forms an oval, that is, since $\Delta_{1}$ is a Galois plane:

THEOREM 4. The set of points $\gamma_{1}=(1,0,0) \cup\left\{\left(h^{2} u, 1, h v\right): h \in F\right\}$ forms a conic $\gamma_{1}$ in a subplane $\Delta_{1}$ of order $q$.

The group of projectivities of the real subplane $\Delta$ which leaves $\gamma$ invariant is doubly-transitive on the vertices of $\gamma$; the extension of the group to $\Omega$ leaves $\Gamma$ invariant and maps $\Delta_{1}$ to other subplanes of order $q$. Consequently, since

$$
\gamma \cap \gamma_{1}=\{(1,0,0),(0,1,0)\}
$$

$\gamma_{1}$ can be mapped to a conic $\gamma_{i}$ which lies in a subplane $\Delta_{i}$, and has among its vertices any two of the vertices of $\gamma$.

In particular, consider the set of $q$ conics $\left\{\gamma_{i}\right\}$ obtained from $\gamma_{1}$ by the set of harmonic homologies of which the axis is a chord of $\gamma$ through $(1,0,0)$ and the vertex is the pole of this chord. If $\left(t^{2}, 1, t\right)$ is the second vertex of $\gamma$ on this chord, then the matrix of the homology is

$$
\left[\begin{array}{ccc}
1 & 4 t^{2} & -4 t \\
0 & 1 & 0 \\
0 & 2 t & -1
\end{array}\right]
$$

and the point $\left(h^{2} u, 1, h v\right)$ maps to

$$
\left((2 t-h v)_{S}^{2}, 1,(2 t-h v)\right) \text { or }\left((2 t-h v)_{N}^{*}(2 t-h v)_{N}, 1,(2 t-h v)\right) \text {. }
$$

Thus every point $\left(\lambda_{S}^{2}, 1, \lambda_{S}\right)$ or $\left(\lambda_{N} \lambda_{N}^{*}, 1, \lambda_{N}^{*}\right)$ of $\Gamma$ belongs to exactly one of these conics. Similarly the subplanes $\Delta_{i}$ into which $\Delta_{1}$ is mapped are disjoint except for the point $(1,0,0)$.

THEOREM 5. The oval $\Gamma$ can be dissected into $q$ conics $\gamma_{i}$ in subplanes $\Delta_{i}$ of order 
$q$, every conic passing through any assigned vertex $K$ of $\gamma$. Each conic $\gamma_{i}$ contains one other vertex of $\gamma$, and, except for $K$, the conics $\gamma_{i}$ are disjoint and the subplanes $\Delta_{i}$ are disjoint.

\section{The polarity determined by the oval $\Gamma$}

There are two known types of polarities in $\Omega$, corresponding to the polarities in the Galois plane determined by a real quadratic form and a Hermitian form (Room, [5]). The numbers of absolute points in the polarities of the two types in $\Omega$ are $\frac{1}{2}\left(q^{3}+q+2\right)$ and $\frac{1}{2}\left(q^{3}+2 q^{2}-q+2\right)$. (An absolute point is a point which is incident with its polar line.) In the paper referred to above, the polarities are the consequence of a combinatorial definition of the Hughes plane; in the present account they are to be defined in terms of the Ostrom coordinates.

In $\Delta$ the conic $\gamma: z^{2}-x y=0$, determines the polarity

$$
(a, b, c) \rightarrow b x+a y-2 c z=0 .
$$

Writing the equation of the line in the form (6)(i) appropriate to $\Omega$ we obtain the mapping:

$$
\left(1,-k, \frac{1}{2}(s-r k)\right) \rightarrow y-s z=k(x-r z) .
$$

We are to show that this polarity can be extended to $\Omega$ in such a way that $\Gamma$ is a subset of the set of absolute points. We prove first:

THEOREM 6. The relations

$$
\begin{gathered}
\mathscr{C}:\left(1,-\kappa, \frac{1}{2}(s-r \kappa)\right) \rightarrow y-s z=\kappa(x-r z), \\
\mathscr{C}^{*}:\left(1,-\kappa, \frac{1}{2}(s-r \kappa)\right) \rightarrow y-s z=\kappa^{*}(x-r z),
\end{gathered}
$$

where $r, s \in F, \kappa \in \Psi$, are polarities in $\Omega$.

The point and line are determined by the triplets of parameters $(\kappa ; r, s)$, so that we may write the mapping $\mathscr{C}$ in the form:

$$
P=P(\kappa ; r, s) \rightarrow \mathscr{C} P=l(\kappa ; r, s) .
$$

We are to prove that

$$
P(\lambda ; a, b) \in l(\kappa ; r, s) \Rightarrow P(\kappa ; r, s) \in l(\lambda ; a, b),
$$

from which relation it follows that a set of collinear points maps to a set of concurrent lines, and that $Q \in \mathscr{C} P \Rightarrow P \in \mathscr{C} Q$. That is, the mapping is a polarity. Also since $P \rightarrow P^{*}$ is an involutory mapping, the mapping $P \rightarrow \mathscr{C} P^{*}=\mathscr{C}^{*} P$ is likewise a polarity.

$$
\begin{aligned}
\left(1,-\lambda, \frac{1}{2}(b-a \lambda)\right) \in y-s z & =\kappa(x-r z) \\
\Rightarrow-\lambda-\frac{1}{2} s(b-a \lambda) & =\kappa\left(1-\frac{1}{2} r(b-a \lambda)\right) .
\end{aligned}
$$


Assume $r a \neq 0$ ( $r=0$ or $a=0$ can easily be dealt with), multiply through by $r a$, and write $\frac{1}{2} r a \lambda=\eta+\frac{1}{2} r b-1$; we obtain

$$
\left(\frac{1}{2} a s-1\right)\left(\eta+\frac{1}{2} b r-1\right)-\frac{1}{4} a b r s=\frac{1}{2} r a \kappa \eta .
$$

Simplifying this relation, and substituting for $\eta$, we find

$$
\left(\frac{1}{2} a s-1-\frac{1}{2} a r \kappa\right)\left(\frac{1}{2} b r-1-\frac{1}{2} a r \lambda\right)=\frac{1}{2} a s+\frac{1}{2} b r-1
$$

This relation is invariant under the interchange $(a, b, \lambda) \leftrightarrow(r, s, \kappa)$, that is, we have the required relation:

$$
P(\lambda ; a, b) \in l(\kappa ; r, s) \Rightarrow P(\kappa ; r, s) \in l(\lambda ; a, b) .
$$

As a preliminary to the investigation of the dependence of these polarities on the oval $\Gamma$, we may state the following connection between ovals and polarities:

THEOREM 7. A given oval in a finite projective plane determines at most one polarity in which all the points of $\Gamma$ are absolute points.

If there is such a polarity, then the polar of each point $P$ of $\Gamma$ is the tangent $t_{P}$ at $P$, the polar of the point which is the intersection of the tangents at $P$ and $Q$ is the chord $P Q$, and, if $K$ is any point of the plane and $P Q, R S$ are two distinct chords through $K$, then the polar of $K$ is $\left\langle t_{P} \cap t_{Q}, t_{R} \cap t_{S}\right\rangle$.

In the Hughes plane $\Omega$ denote by $\mathbb{E}$ and $\mathbb{E} *$ the sets of absolute points in the polarities $\mathscr{C}$ and $\mathscr{C}^{*}$, that is, $\mathbb{C}=\{P: P \in \mathscr{C} P\}$ and $\mathbb{C}^{*}=\left\{P: P \in \mathscr{C}^{*} P\right\}$. Both $\mathbb{E}$ and $\mathfrak{C}^{*}$ include the set of points $\gamma$ in the subplane $\Delta$. If we write the coordinates of a point of $\Omega$ as $(1, \alpha, \beta)$, so that its polar line in $\mathscr{C}$ is

where

$$
y-s z+\alpha(1-r z)=0,
$$

$$
\alpha=a+v a^{\prime}, \quad \beta=b+v b^{\prime}, \quad 2 \beta=s+r \alpha,
$$

so that

$$
2 b=s+r a, 2 b^{\prime}=r^{\prime} a^{\prime},
$$

then,

$$
\begin{aligned}
\mathbb{C} & =\{(1, \alpha, \beta): \alpha-s \beta+\alpha(1-r \beta)=0 \text { in } \Psi\} \cup(0,1,0), \\
\mathfrak{C}^{*} & =\left\{(1, \alpha, \beta): \alpha-s \beta+\alpha^{*}(1-r \beta)=0 \text { in } \Psi\right\} \cup(0,1,0) .
\end{aligned}
$$

It follows that, formally,

$$
\mathfrak{C} \cup \mathfrak{C}^{*}=\Gamma_{\Phi} \cup \Gamma_{\Phi}^{*}
$$

where $\Gamma_{\Phi}$ is the conic $x y=z^{2}$ in the Galois plane over $\Phi$, and $\Gamma_{\Phi}^{*}$ is the corresponding Hermitian set of $q^{3}+1$ absolute points.

The sets $\mathbb{C}$ and $\mathbb{C}^{*}$ are to be split into subsets $\mathbb{C}_{S}, \mathbb{C}_{N}, \mathbb{C}_{S}^{*}, \mathbb{C}_{N}^{*}$, where

$$
\mathfrak{C}_{s}=\left\{(1, \alpha, \beta): \alpha-s \beta+\alpha(1-r \beta)=0 \text { in } \Psi \& 1-r \beta \in \Phi_{S}\right\} \text {, etc. }
$$

It is to be proved that: 
THEOREM 8. The constituent parts of the sets of absolute points for the polarities $\mathfrak{E}=\mathfrak{S}_{S} \cup \mathfrak{S}_{N}$ and $\mathfrak{C}^{*}=\mathfrak{S}_{S}^{*} \cup \mathfrak{C}_{N}^{*}$ are

$$
\mathfrak{C}_{S}^{*} \supset \gamma \text { and }\left|\mathfrak{C}_{S}-\gamma\right|=\frac{1}{2}\left(q^{3}-q\right),\left|\mathfrak{C}_{N}\right|=\frac{1}{2}\left(q^{3}-q\right) ;
$$

for $q \equiv-1(\bmod 4)$ :

$$
\mathfrak{C}_{S}=\Gamma, \mathbb{C}_{N}^{*}=\emptyset
$$

for $q \equiv 1(\bmod 4)$ :

$$
\mathfrak{C}_{S}=\gamma, \quad \mathfrak{C}_{N}^{*}=\Gamma-\gamma .
$$

To convert the coordinates of the points of $\Gamma$ to the form in which the first coordinate is 1 , replace by $\tau^{-1}$ the parameter $\rho$ in the forms $\left(\rho_{S}, \rho_{S}^{-1}, 1\right)$, $\left(\rho_{N}, \rho_{N}^{*-1}, 1\right)$ used in $\S 2$, so that the new parametric forms are $\left(1, \tau_{S}^{2}, \tau_{S}\right)$, $\left(1, \tau_{N}^{*} \tau_{N}, \tau_{N}\right)$ in $\Psi$. We are to carry out algebraic operations in $\Phi$ rather than in $\Psi$, so that we may treat the two forms of coordinates together, by writing $\Gamma=$ $\left\{(1, \omega, \tau): \omega=\left(\tau^{2}\right)_{\Phi}\right\} \cup(0,1,0)$.

Now consider simultaneously the two sets of points:

$$
\mathbb{C}_{s}, \mathbb{C}_{N}^{*}=\{(1, \alpha, \beta) ; \alpha-s \beta+\alpha(1-r \beta)=0 \text { in } \Phi\},
$$

where, for $\mathfrak{C}_{s}, 1-r \beta \in \Phi_{S}$ and, for $\mathfrak{C}_{N}^{*}, 1-r \beta \in \Phi_{N}$.

The condition satisfied by the points $(1, \alpha, \beta)$ reduces to

that is

$$
\alpha=\left(\beta^{2}\right)_{\Phi},
$$

so that

$$
a=b^{2}+u b^{\prime 2}, a^{\prime}=2 b b^{\prime}
$$

$$
1-r \beta=-v \cdot 2 b^{\prime 2} / a^{\prime} .
$$

Consequently (excluding the set for which $\beta \in F$ ), we have

$$
1-r \beta \in \Phi_{\mathrm{S}}-F \Rightarrow-u \in F_{\mathrm{S}} \Rightarrow-1 \in F_{N} .
$$

Thus, the condition $1-r \beta \in \Phi_{S}$ is a restriction not on the choice of $\beta$ in the coordinates $\left(1,\left(\beta^{2}\right)_{\Phi}, \beta\right)$, but on the characteristic of the Galois field itself. That is, when, in $G F(q),-1 \in F_{N}$, every point $(1, \alpha, \beta)$ of $\mathbb{E}_{S}$ satisfies the condition $\alpha=\left(\beta^{2}\right)_{\Phi}$, and therefore $\mathfrak{夭}_{S}=\Gamma$, while no point of $\mathfrak{E}_{N}$ satisfies the condition. Likewise when, in $G F(q),-1 \in F_{S}, \mathfrak{C}_{N}^{*}=\Gamma-\gamma$ and, as is to be proved later, $\mathfrak{C}_{S}^{*} \supset \gamma$.

Let us consider now the other two sets:

$$
\mathfrak{C}_{N}, \mathbb{\complement}_{S}^{*}=\left\{(1, \alpha, \beta): \alpha-s \beta+\alpha^{*}(1-r \beta)=0 \text { in } \Phi\right\}
$$

The condition satisfied by the points of the sets is

that is,

$$
2 \alpha+\alpha^{*}-\left(\beta^{*} \beta\right)_{\Phi}=0,
$$

$$
2 a=b^{2}-u b^{\prime 2}=d_{\beta} .
$$

Thus $\mathfrak{C}_{N}, \mathbb{C}_{S}^{*}$ are the subsets of the set of points $\left\{\left(1, d_{\beta}+v a^{\prime}, \beta\right)\right\}$ for which $1-r \beta$ belongs respectively to $\Phi_{N}$ and $\Phi_{S}$. We have 


$$
a^{\prime 2} d_{1-r \beta}=\left(a^{\prime}-a^{\prime} r b\right)^{2}-a^{\prime 2} u r^{2} b^{\prime 2}=\left(a^{\prime}-2 b b^{\prime}\right)^{2}-4 u b^{\prime 4} .
$$

Thus, to obtain the set $\mathbb{E}_{N}$, the parameter $\beta$ in the coordinates $\left(1, d_{\beta}+v a^{\prime}, \beta\right)$ can be selected arbitrarily in $\Psi$, while $a^{\prime}$ has to be such that $\left(a^{\prime}-2 b b^{\prime}\right)^{2}-4 u b^{\prime 4} \in F_{N}$. For $\left(_{S}^{*}\right.$ we have $\left(a^{\prime}-2 b b^{\prime}\right)^{2}-4 u b^{\prime 4} \in F_{S}$. This condition is satisfied for all $a^{\prime}$ when $b^{\prime}=0$, and $\mathfrak{C}_{S}^{*}$ therefore contains the set of points $\left\{\left(1, b^{2}-v a^{\prime}, b\right): a^{\prime}, b \in F\right\}$; $\gamma$ is the subset of this set for which $a^{\prime}=0$. The explicit listing of the sets of absolute points in the combinatorial description of $\Omega$ (Room [5]) shows that, in agreement with the numbers of solutions of the above congruences, each of the sets $\mathfrak{S}_{N}$ and $\mathfrak{C}_{\mathrm{S}}^{*}-\gamma$ consists of $\frac{1}{2}\left(q^{3}-q\right)$ points.

\section{References}

[1] D. R. Hughes, 'A class of non-Desarguesian projective planes', Can. J. Math. 9 (1957), $378-388$.

[2] Marshall Hall, The theory of groups (New York, Macmillan, 1959) p. 416.

[3] T. G. Ostrom, 'A class of non-Desarguesian affine planes', Trans. Am. Math. Soc. 104 (1962), 483-487.

[4] L. A. Rosati, 'I gruppi di collineazioni dei piani di Highes', Bull. Un. Mat. Ital. 13 (1958), 505-513.

[5] T. G. Room, 'The combinatoral structure of the Hughes plane', Proc. Camb. Phil. Soc., phys. math. sci. 68 (1970) 291-301.

The Open University

Walton Hall, Bletchley

Bucks, V.K. 